\title{
Characterization of Poly(methyl methacrylate) Prepared by Plasma-Initiated Polymerization
}

\author{
Yasuhiro Kashiwagi, Yoshiyuki EINAGA, and Hiroshi FuJITA \\ Department of Polymer Science, Osaka University, \\ Toyonaka, Osaka 560, Japan.
}

(Received December 28, 1979)

KEY WORDS Plasma-initiated polymerization / Radius of Gyration-
Molecular Weight Relation / Intrinsic Viscosity-Molecular Weight Relation /

\begin{abstract}
Recently, Osada et al. ${ }^{1}$ found that an ultrahigh molecular weight sample of poly(methyl methacrylate)(PMMA) can be readily prepared by the plasma-initiated polymerization technique. In response to the request of the late Professor M. Shen of the University of California, Berkeley, California, we have attempted a light scattering and viscometric characterization of three PMMA samples newly prepared by this technique and put at our disposal. The main concern was to estimate the absolute molecular weights, heterogeneity, and linearity of these samples. The results obtained are described briefly in this note.
\end{abstract}

\section{EXPERIMENTAL}

\section{Samples and Solution}

The three samples were designated as 003,178 , and 194, according to the sender's indication. They were subjected to light scattering and viscosity measurements with methyl ethyl ketone (MEK) at $25^{\circ} \mathrm{C}$ as the solvent. Sample 194 was also examined in acetone at $25^{\circ} \mathrm{C}$.

Solubility tests with acetone indicated that sample 003 contained a substantial amount of insoluble fraction. This fraction was removed by centrifugation, and the polymer was precipitated from the supernatant by the addition of methanol, freezedried from a benzene solution, and further dried under reduced pressure. Samples 178 and 194 were directly precipitated from acetone solutions with methanol and dried under reduced pressure.

Each of these purified samples was dissolved in either acetone or MEK without stirring to prevent any shear degradation; the polymer-solvent mixture was left standing at room temperature for 5 to 7 days and then shaken gently for about half a day to ensure complete dissolution. The solution so prepared was diluted with the respective solvent to a desired concentration one day before each measurement. The solvents used were purified thoroughly by standard procedures.

\section{Light-Scattering Measurement}

A Fica 50 light-scattering photogoniometer was used, with vertically polarized light of $546 \mathrm{~nm}$ wavelength as the incident beam. Each test solution or the solvent was made optically clean by centrifuging for about $100 \mathrm{~min}$ at $11,000 \mathrm{~g}$ in a Sorvall RC2-B centrifuge and transferred directly into the light scattering cell using a pipet. Both the cell and pipet had been rinsed with acetone vapor for more than $6 \mathrm{~h}$ and completely dried.

Intensities of scattered light were measured at intervals of $2.5^{\circ}$ in the range of scattering angle from $15^{\circ}$ to $40^{\circ}$ and at intervals of $15^{\circ}$ in the range from $45^{\circ}$ to $150^{\circ}$. No reliable data could be obtained at scattering angles below $15^{\circ}$. The cell constant was determined by the usual method, assuming $16.5 \times 10^{-6} \mathrm{~cm}^{-1}$ for the Rayleigh ratio of pure benzene for unpolarized light of $546 \mathrm{~nm}$ and using 0.41 obtained for the depolarization ratio of benzene.

The values 0.111 and $0.1293 \mathrm{~cm}^{3} \mathrm{~g}^{-1}$ were taken from the literature ${ }^{2}$ for the specific refractive index increments of PMMA in MEK at $25^{\circ} \mathrm{C}$ and in 
acetone at $25^{\circ} \mathrm{C}$, respectively.

\section{Viscometery}

In view of the expected high molecular weights of the samples, the rotational viscometer of the ZimmCrothers type described elsewhere ${ }^{3}$ was used, and the measurements were carried out at shear rates of about $7 \mathrm{~s}^{-1}$. These shear rates were chosen on the basis of preliminary experiments indicating that the viscosities of our solutions were essentially independent of the shear rate $G$, if $G$ was less than $25 \mathrm{~s}^{-1}$.

\section{RESULTS AND DISCUSSION}

\section{Light-Scattering Data}

Figure 1 illustrates the angular variations of $\left(K c / R_{\theta}\right)^{1 / 2}$ at five polymer mass concentrations $c$, for sample 178 in $\mathrm{MEK}$ at $25^{\circ} \mathrm{C}$. Here, $K$ is the familiar optical constant and $R_{\theta}$ is the reduced intensity of scattered light at the scattering angle $\theta$. Similar data were obtained for samples 003 and 194 in MEK and for sample 194 in acetone. In Figure 2, the data of Figure 1 are replotted as $K c / R_{\theta}$ against $c$ for fixed values of $\theta$. The set of data points for each $\theta$ is fitted

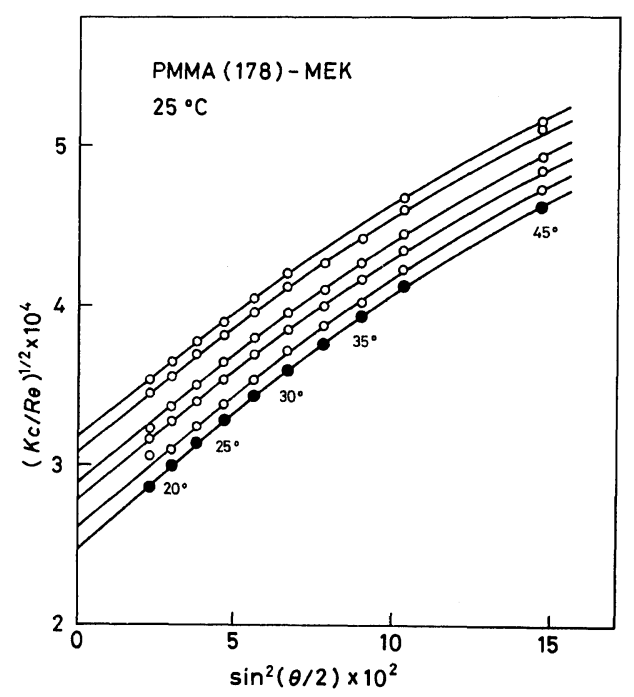

Figure 1. Angular dependence of reciprocal squareroot of reduced scattering intensity for sample 178 in MEK at $25^{\circ} \mathrm{C}$. Polymer concentrations are $2.08_{5} \times 10^{-4}$, $1.66_{5} \times 10^{-4}, 1.23_{1} \times 10^{-4}, 0.84_{2} \times 10^{-4}$, and $0.43_{3} \times 10^{-4}$ $\mathrm{g} \mathrm{cm}^{-3}$ from top to bottom. Filled circles represent the values extrapolated to zero concentration.

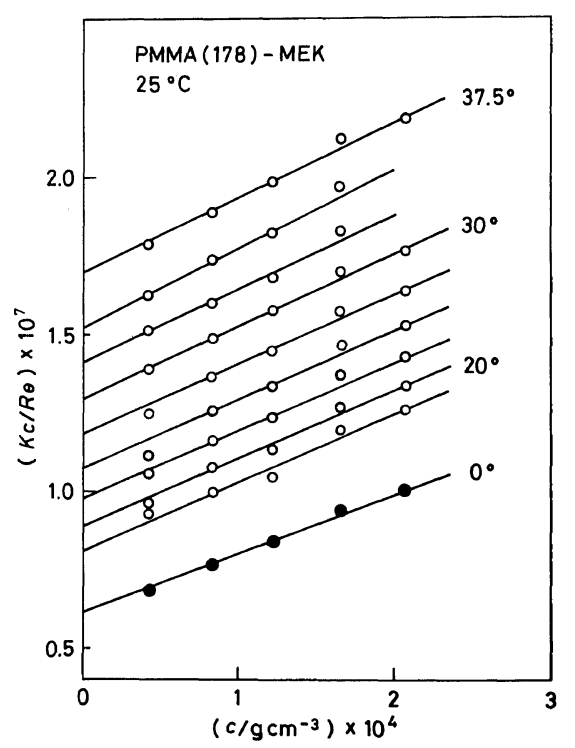

Figure 2. Reciprocal reduced scattering intensity at indicated angles as a function of polymer concentration for sample 178 in $\mathrm{MEK}$ at $25^{\circ} \mathrm{C}$. Filled circles represent the values extrapolated to zero scattering angle.

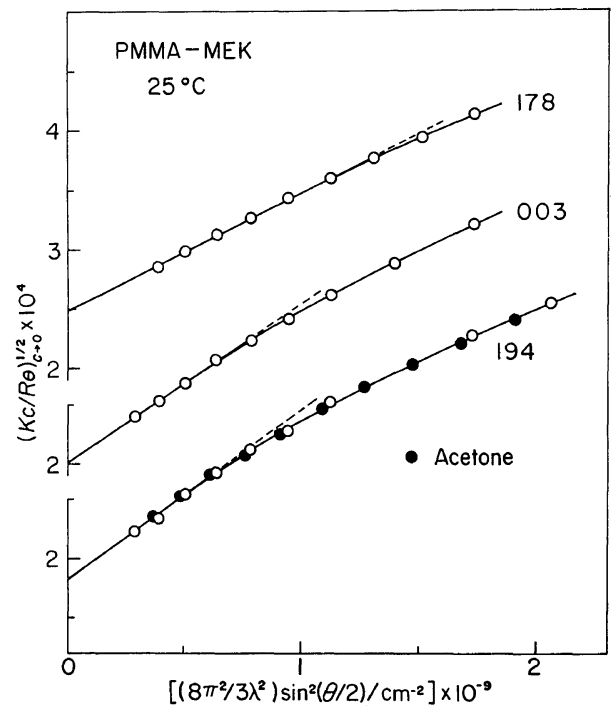

Figure 3. Reciprocal square-root of reduced scattering intensity at zero concentration as a function of scattering angle for three PMMA samples in MEK (unfilled circles) and for sample 194 in acetone (filled circles) at $25^{\circ} \mathrm{C}$. 
Table I. Numerical results from light scattering measurements on PMMA in MEK and acetone at $25^{\circ} \mathrm{C}$

\begin{tabular}{|c|c|c|c|c|}
\hline \multirow{2}{*}{$\begin{array}{l}\text { Sample } \\
\text { code }\end{array}$} & \multirow{2}{*}{ Solvent } & \multirow{2}{*}{$M_{w} \times 10^{-7^{*}}$} & $A_{2} \times 10^{5}$ & $\left\langle S^{2}\right\rangle_{z}^{1 / 2}$ \\
\hline & & & $\mathrm{cm}^{3} \mathrm{~mol} \mathrm{~g}^{-2}$ & $\mathrm{~nm}$ \\
\hline 178 & MEK & $1.61 \pm 0.05$ & $8.6 \pm 0.3$ & $199 \pm 5$ \\
\hline 003 & MEK & $2.45 \pm 0.07$ & $7.5 \pm 0.3$ & $257 \pm 7$ \\
\hline 194 & MEK & $3.00 \pm 0.1$ & $7.0 \pm 0.7$ & $280 \pm 10$ \\
\hline 194 & Acetone & $3.00 \pm 0.1$ & $6.0 \pm 0.5$ & $280 \pm 10$ \\
\hline
\end{tabular}

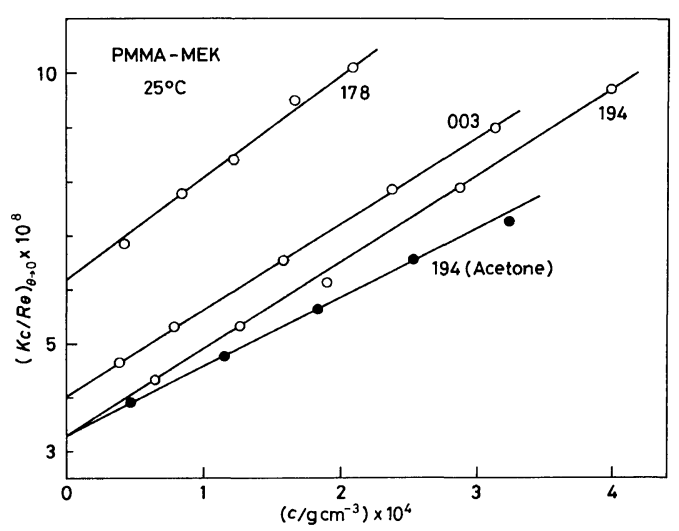

Figure 4. Reciprocal reduced scattering intensity at the zero scattering angle as a function of polymer concentration for three PMMA samples in MEK (unfilled circles) and for sample 194 in acetone (filled circles) at $25^{\circ} \mathrm{C}$.

by a straight line, permitting the infinite-dilution value of $K c / R_{\theta}$ to be estimated accurately. The filled circles in Figure 1 show the square-root of these infinite-dilution values as a function of $\sin ^{2}(\theta / 2)$. On the other hand, the filled circles in Figure 2 show the concentration dependence of the zero-angle values of $K c / R_{\theta}$ extrapolated from the solid curves in Figure 1.

Figures 3 and 4 display the angular dependence of $\left(K c / R_{\theta}\right)_{c=0}^{1 / 2}$ and the concentration dependence of $\left(K c / R_{\theta}\right)_{\theta=0}$ for the four systems. Table I summarizes the values of the weight-average molecular weight $M_{w}$, the root-mean-square radius of gyration $\left\langle S^{2}\right\rangle^{1 / 2}$, and the second virial coefficient $A_{2}$ determined from these data. As was expected, essentially the same values were obtained for the weightaverage molecular weights of sample 194 in MEK and in acetone. This agreement implies that the polymer in these solvents is dispersed molecularly.

The feature of the plasma-initiated polymerization technique, i.e., that it can yield a very high molecular weight, a property normally difficult to obtain by conventional initiation methods, is evident from the $M_{w}$ values presented in Table I. Probably, the $M_{w}$ value of sample 194 is the highest absolute molecular weight so far reported for PMMA.

In Figure 5 are shown the values of $x P(x)$ for the three samples in MEK as a function of $x^{1 / 2}$, where $x$ is defined by $x=(4 \pi / \lambda)^{2}\left\langle S^{2}\right\rangle^{1 / 2} \sin ^{2}(\theta / 2)$, with $\lambda$ being the wavelength of the incident beam in the solution and $P(x)$ is the particle scattering function. The discrepancy of the plotted points from the Debye function indicated by the solid line suggests that these samples are not as homogeneous in

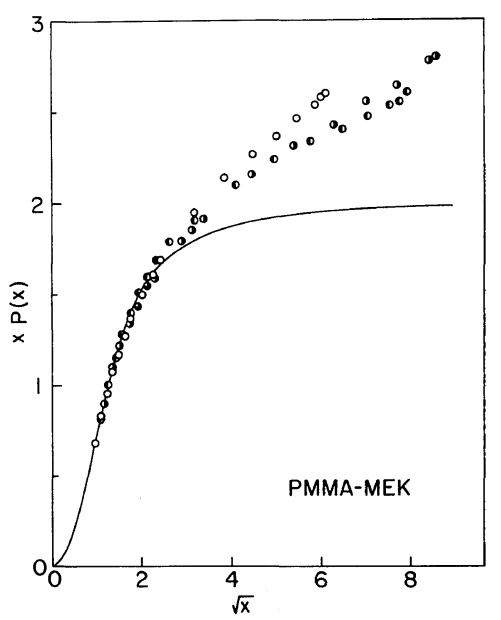

Figure 5. Particle scattering functions $P(\theta)$ represented as a plot of $x P(x)$ vs. $x^{1 / 2}$ for samples $178(O), 003(D)$, and 194 ( $)$ in $\mathrm{MEK}$ at $25^{\circ} \mathrm{C}$. The solid line represents the Debye function for a monodisperse Gaussian coil. 
molecular weight as expected originally. ${ }^{1}$

\section{Viscosity Data}

Figure 6 shows the usual viscosity plots for the four systems studied. We note that these data refer essentially to the zero shear rate. Table II gives the values of intrinsic viscosity $[\eta]$ and the Huggins constant $k^{\prime}$ estimated from Figure 6 . The $k^{\prime}$ values are normal, suggesting that our high-molecularweight PMMA samples dispersed molecularly in both MEK and acetone.

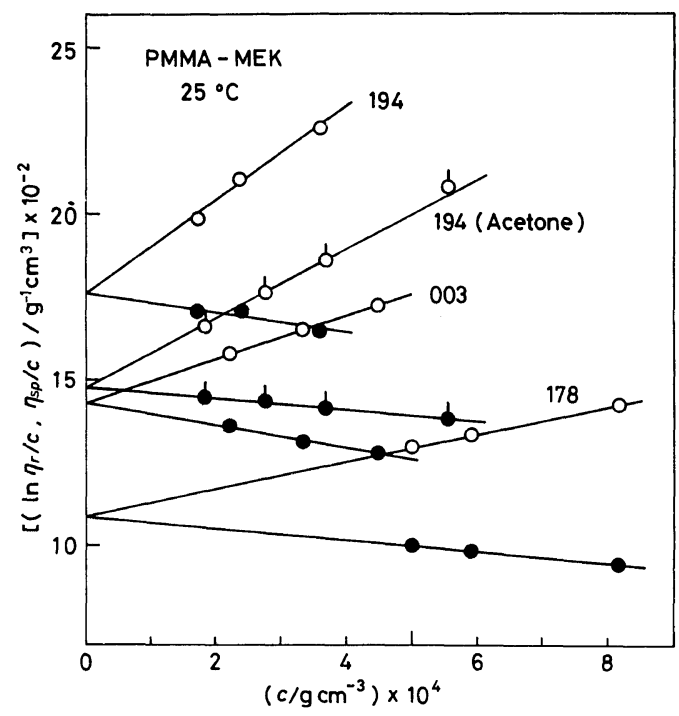

Figure 6. Concentration dependence of $\eta_{\text {sp }} / c$ (unfilled circles) and $\ln \eta_{\mathrm{r}} / c$ (filled circles) at the zero shear rate for three PMMA samples in MEK (without pip) and for sample 194 in acetone (with pip) at $25^{\circ} \mathrm{C}$.

$$
\begin{aligned}
& {[\eta]=(1.1 \pm 0.2) \times 10^{-2} M_{w}^{0.69 \pm 0.01}} \\
& \left.[\eta]=(5.7 \pm 0.2) \times 10^{-3} M_{w}^{0.70 \pm 0.01} \text { at } 25^{\circ} \mathrm{C}\right)
\end{aligned}
$$

(Acetone at $25^{\circ} \mathrm{C}$ )

Table II. Numerical results from viscosity measurements on PMMA in MEK and acetone at $25^{\circ} \mathrm{C}$

\begin{tabular}{llll}
\hline \multirow{2}{*}{$\begin{array}{c}\text { Sample } \\
\text { code }\end{array}$} & Solvent & \multirow{2}{*}{$k^{\prime}$} \\
\cline { 3 - 3 } & & $\mathrm{cm}^{3} \mathrm{~g}^{-1}$ & \\
\hline 178 & MEK & $10.9 \pm 0.1$ & 0.34 \\
003 & MEK & $14.3 \pm 0.2$ & 0.33 \\
194 & MEK & $17.6 \pm 0.3$ & 0.44 \\
194 & Acetone & $14.8 \pm 0.2$ & 0.47 \\
\hline
\end{tabular}

Acknowledgment. We should like to dedicate this paper to the late Professor M. Shen of the University of California, Berkeley, in the memory of his very kind friendship toward all of us. The interest of Dr. Y. Osada of Ibaraki University in this work is gratefully acknowledged.

\section{REFERENCES}

1. Y. Osada, A. T. Bell, and M. Shen, Polym. Lett., 16, 309 (1978). 


\section{Characterization of PMMA}

2. M. G. Huglin, "Light Scattering from Polymer Solutions," Academic Press, London and New York, N.Y., 1972.

3. Y. Einaga, Y. Miyaki, and H. Fujita, J. Soc. Rheol. Jpn., 5, 188 (1977).

4. S. N. Chinai, J. D. Matlack, A. L. Resnick, and R. J. Samuels, J. Polym. Sci., 17, 391 (1955).
5. S. N. Chinai and C. W. Bondurant, Jr., J. Polym. Sci., 22, 555 (1956).

6. G. V. Schulz, H.-J. Cantow, and G. Meyerhoff, $J$. Polym. Sci., 10, 79 (1953).

7. E. Cohen-Ginsburg, T. G Fox, and H. F. Mason, Polymer, 3, 97 (1962). 